\title{
BMJ Open Methodological review to develop a list of bias items used to assess reviews incorporating network meta-analysis: protocol and rationale
}

\author{
Carole Lunny (D) , ${ }^{1}$ Andrea C Tricco (D) , , ${ }^{1,2,3}$ Areti-Angeliki Veroniki, ${ }^{4}$ Sofia Dias (D) ${ }^{5}$ \\ Brian Hutton (D) , ${ }^{6,7}$ Georgia Salanti, ${ }^{8}$ James M Wright, ${ }^{9}$ lan White, ${ }^{10}$ \\ Penny Whiting ${ }^{11}$
}

To cite: Lunny C, Tricco AC, Veroniki A-A, et al. Methodological review to develop a list of bias items used to assess reviews incorporating network meta-analysis: protocol and rationale. BMJ Open 2021;11:e045987. doi:10.1136/ bmjopen-2020-045987

- Prepublication history and additional supplemental material for this paper are available online. To view these files, please visit the journal online (http://dx.doi.org/10.1136/ bmjopen-2020-045987)

Received 21 October 2020 Accepted 07 June 2021

Check for updates

(C) Author(s) (or their employer(s)) 2021. Re-use permitted under CC BY-NC. No commercial re-use. See rights and permissions. Published by BMJ.

For numbered affiliations see end of article.

Correspondence to

Carole Lunny;

carole.lunny@ubc.ca

\section{ABSTRACT}

Introduction Systematic reviews with network metaanalysis (NMA; ie, multiple treatment comparisons, indirect comparisons) have gained popularity and grown in number due to their ability to provide comparative effectiveness of multiple treatments for the same condition. The methodological review aims to develop a list of items relating to biases in reviews with NMA. Such a list will inform a new tool to assess the risk of bias in NMAs, and potentially other reporting or quality checklists for NMAs which are being updated.

Methods and analysis We will include articles that present items related to bias, reporting or methodological quality, articles assessing the methodological quality of reviews with NMA, or papers presenting methods for NMAs. We will search Ovid MEDLINE, the Cochrane library and difficult to locate/unpublished literature. Once all items have been extracted, we will combine conceptually similar items, classifying them as referring to bias or to other aspects of quality (eg, reporting). When relevant, reporting items will be reworded into items related to bias in NMA review conclusions, and then reworded as signalling questions.

Ethics and dissemination No ethics approval was required. We plan to publish the full study open access in a peer-reviewed journal, and disseminate the findings via social media (Twitter, Facebook and author affiliated websites). Patients, healthcare providers and policymakers need the highest quality evidence to make decisions about which treatments should be used in healthcare practice. Being able to critically appraise the findings of systematic reviews that include NMA is central to informed decision-making in patient care.

\section{INTRODUCTION}

Reviews with network meta-analysis (NMAs) have gained popularity due to their ability to provide comparative effectiveness of multiple treatments for the same condition. ${ }^{1}$ Reviews with NMA have grown in number. Between 1997 and 2015, 771 NMAs were published in 336 journals from 3459 authors and 1258 institutions in 49 countries. $^{2}$ More
Strengths and limitations of this study

- No tool for assessment of biases in reviews with network meta-analysis (NMA) currently exists.

- Our research aims to develop a list of items related to bias in the goal of developing the first tool for assessing risk of bias in the findings of NMAs.

- A comprehensive and systematic process will be followed to develop a risk of bias tool for assessing reviews with NMAs, as outlined in Whiting et a/s 'Framework for Developing Quality Assessment Tools', starting with this methodological review to develop a list of bias items used to assess NMAs

- One limitation is that the items identified through this methodological review should be considered as possible contenders for inclusion in the risk of bias in NMAs tool since items have not been vetted through a Delphi exercise with experts as of yet.

- Wording of the items may change after conducting the Delphi and pilot testing exercises.

than three-quarters $(n=625 ; 81 \%)$ of these NMAs were published in the last 5 years. Many organisations such as the National Institute for Health and Care Excellence (NICE) in the UK, the World Health Organisation (WHO) and the Canadian Agency for Drugs and Technologies in Health (CADTH) conduct NMAs as they represent the best available evidence to inform clinical practice guidelines. ${ }^{3-5}$ We adopt a broad definition of NMAs, specifically: a review that aims to, or intends to, simultaneously synthesise more than two heath care interventions of interest. Reviews that intend to compare multiple treatments with an NMA but then find that the assumptions are violated (eg, a disconnected network, or studies are too heterogeneous to combine) and that NMA is not feasible, will also be included in our definition.

Evidence shows that biased results from poorly designed and reported studies can 
mislead decision-making in healthcare at all levels. ${ }^{6-9}$ If a review is at risk of bias and inappropriate methods are used, the validity of the findings can be compromised. ${ }^{10-12}$ Evaluating how well a review has been conducted is essential to determining whether the findings are relevant to patient care and outcomes. Several empirical studies have shown that bias can obscure the real effects of a treatment. ${ }^{13-16}$ Being able to appraise reviews with NMA is central to informed decision-making in patient care.

The systematic procedures required to conduct a systematic review help mitigate the risk of bias. However, bias can also be introduced when interpreting the reviews findings. For example, review's conclusions may not be supported by the evidence presented, the relevance of the included studies may not have been considered by review authors, and reviewers may inappropriately emphasise results on the basis of their statistical significance. ${ }^{17}$ A wellconducted systematic review draws conclusions that are appropriate to the included evidence and can therefore be free of bias even when the primary studies included in the review have high risk of bias.

Tools are available for most study designs to make risk of bias assessment easier for a knowledge user (eg, healthcare practitioners, policymakers, patients ${ }^{18}$ ). Many tools and checklists can be used either when conducting a systematic review (quality of conduct), when assessing how well a study has been described (reporting), or when knowledge users want to assess the risk of bias in the conclusions of a review. The methodological quality of studies (ie, how well the study is conducted) is often confused with reporting quality (ie, how well authors describe their methodology and results). A risk of bias assessment is an assessment of review limitations, which focus on the potential of those methods to bias the study findings. ${ }^{17}$

More than 40 tools have been identified ${ }^{1920}$ for critically appraising the quality of reviews with pairwise metaanalysis. AMSTAR (A MeaSurement Tool to Assess the methodological quality of systematic Reviews $)^{21}$ and the OQAQ (Overview Quality Assessment Questionnaire ${ }^{22}$ ) have been identified as the most commonly used, and they follow a simple checklist format. ${ }^{20}$ AMSTAR has been recently updated to AMSTAR 2, which aims to evaluate how reviews are planned and conducted. ${ }^{24}$ The ROBIS (Risk Of Bias In Systematic reviews) tool is designed to assess the risk of bias in systematic reviews with or without pairwise meta-analysis. ${ }^{17}$ The ROBIS tool involves assessment of methodological features in reviews known to increase the risk of bias in review conclusions. Domain-based assessment tools require a careful reading and thoughtful analysis of the study to adequately rate risk of bias, instead of simply identifying keywords reported in the article, as usually made in a checklist type of assessment.

For critically appraising reviews with NMA, several checklists exist. To assess reporting quality, the Preferred Reporting Items for Systematic Reviews and Meta-Analyses statement extension for reviews incorporating network meta-analysis (PRISMA-NMA) ${ }^{25}$ or the National Institute for Health and Care Excellence Decision Support Unit checklist (NICE-DSU) ${ }^{26}$ can be used. To assess quality of conduct, the International Society for Pharmacoeconomics and Outcomes Research (ISPOR) checklist ${ }^{27}$ can be used. However, many quality assessment tools are not created rigorously. To be rigorous, they must follow a series of systematic steps. ${ }^{28} 29$ As a quality of conduct tool, the ISPOR checklist ${ }^{27}$ did not follow the methodology proposed by Whiting ${ }^{29}$ for creating a systematically developed quality tool. Due to important methodological advances in the field of NMA, the ISPOR, published in 2014, is also outdated. As table 1 shows, several tools are designed with different purposes; some for assessing reporting quality, and some for assessing quality of conduct but none are designed to assess risk of bias in NMAs.

A comprehensive and systematic process should be used to develop a rigorous risk of bias tool for assessing NMAs, as outlined in Whiting et al $\mathrm{s}^{29}$ 'Framework for Developing Quality Assessment Tools' The first step is to: (1) conduct a systematic search of biases that can inform the assessment of the validity and reliability of NMAs and prepare a pilot list of items, (2) create a draft tool, (3) obtain expert opinion on the draft tool and inclusion of items through Delphi exercises, and (4) pilot test and refine the tool. ${ }^{29}$ No review has comprehensively and systematically listed and categorised all items related to bias in NMAs. Such a list will inform a new tool to assess the risk of bias in NMAs, and potentially other reporting or quality tools which are being updated.

\section{OBJECTIVE}

Our objective for this protocol paper is to plan the conduct of a methodological review to develop a list of items relating to bias in NMAs. This is the first step in the goal of developing a risk of bias tool to assess NMAs. Further steps will involve conducting a series of Delphi surveys to select, refine and compile items into a tool; pilot test and then refine the draft tool with different user groups; and finally develop and evaluate an evidencebased (knowledge translation) KT strategy to disseminate the tool. This protocol pertains to our first objective to systematically search for and identify a list of bias items for NMAs.

\section{METHODS AND ANALYSIS}

We will follow the methodology proposed by Whiting, ${ }^{29}$ Sanderson ${ }^{30}$ and Page ${ }^{7}$ for creating systematically developed lists of quality items. Although this protocol is for a methodological review, and not a health intervention review, our protocol was described and reported in accordance with the preferred reporting items for systematic reviews and meta-analysis protocol (PRISMA-P) checklist with not applicable indicated for items not pertaining to methods reviews ${ }^{31}$ (online supplemental appendix 1). 
Table 1 Tools and checklists to aid in systematic review conduct, or to assess the reporting or methodological quality of a review

\begin{tabular}{|c|c|c|c|}
\hline Tool purpose & $\begin{array}{l}\text { Examples } \\
\text { of tools or } \\
\text { checklists }\end{array}$ & Description of the example checklist or tool & $\begin{array}{l}\text { Available tool for } \\
\text { reviews with NMA }\end{array}$ \\
\hline $\begin{array}{l}\text { Guidance for } \\
\text { conducting } \\
\text { systematic reviews }\end{array}$ & $\mathrm{MECIR}^{34}$ & $\begin{array}{l}\text { Detailed methodological guidance on how to conduct a systematic } \\
\text { review with or without pairwise meta-analysis of effectiveness, } \\
\text { diagnostic test accuracy, individual patient data reviews and } \\
\text { reviews in public health and health promotion }\end{array}$ & No \\
\hline $\begin{array}{l}\text { Assess the risk of } \\
\text { bias of published } \\
\text { reviews }\end{array}$ & $\mathrm{ROBIS}^{17}$ & $\begin{array}{l}\text { ROBIS is a tool for assessing the risk of bias in reviews. It is } \\
\text { aimed at four broad categories of reviews mainly within healthcare } \\
\text { settings: interventions, diagnosis, prognosis, and aetiology. }\end{array}$ & No \\
\hline $\begin{array}{l}\text { Assess the certainty } \\
\text { in evidence and } \\
\text { the strength of } \\
\text { recommendations }\end{array}$ & GRADE $^{39}$ & $\begin{array}{l}\text { GRADE approach defines the certainty of a body of evidence as } \\
\text { the extent to which one can be confident that a pooled effect } \\
\text { estimate is close to the true effect of the intervention. Five domains } \\
\text { assessed: risk of bias, inconsistency, indirectness, imprecision and } \\
\text { publication bias. }\end{array}$ & $\begin{array}{l}\text { GRADE-NMA, } \\
{ }^{41} \text { CINeMA, } \\
\text { Threshold method }^{43}\end{array}$ \\
\hline
\end{tabular}

AMSTAR-2, A Measurement Tool to Assess Systematic Reviews 2; CINeMA, Confidence in Network Meta-Analysis; GRADE, Grading of Recommendations Assessment, Development and Evaluation; GRADE-NMA, Grading of Recommendations Assessment, Development and Evaluation for Network Meta-Analysis; ISPOR, International Society for Pharmacoeconomics and Outcomes Research; MECIR, Methodological Expectations of Cochrane Intervention Reviews; NICE-DSU, National Institute for Health and Care Excellence Decision Support Unit checklist; OQAQ, Overview Quality Assessment Questionnaire; PRISMA, Preferred Reporting Items for Systematic Reviews and Meta-Analyses; PRISMA-NMA, PRISMA statement extension for reviews incorporating NMA; ROBIS, Risk Of Bias In Systematic reviews.

\section{Eligibility criteria}

There will be two types of studies included. Study type 1 are articles that present and describe items related to bias, reporting or methodological quality of reviews with NMA. Items related to reporting will be retained because they can potentially be translated into a risk of bias item. For example, in the PRISMA-P guideline, ${ }^{31}$ one item asks whether study PICO (Population, Interventions, Comparisons, Outcomes) characteristics were used as criteria for determining study eligibility. Reporting of all outcomes in a protocol may prevent authors from only selecting outcomes that are statistically significant when publishing their systematic review. This PRISMA-P reporting item can then be translated into a bias item related to the 'selective reporting' of outcomes. ${ }^{32}$ Study type 2 are studies that assess the methodological quality in a sample of reviews with NMA.

\section{Study type 1 will meet any of these inclusion criterion}

- Articles describing items related to bias or methodological quality in reviews with NMA (eg, Dias 2018 ${ }^{33}$ ); tools that only assess general aspects of systematic reviews without focusing specifically on NMA will be excluded (eg, AMSTAR, ${ }^{21}$ AMSTAR $2^{24}$ or ROBIS ${ }^{17}$ ).

- Articles describing editorial standards for reviews with NMA (eg, similar to the Cochrane MeCIR (Methodological standards for the conduct of new Cochrane
Intervention Reviews) standards for systematic reviews ${ }^{34}$ ).

- Articles describing items related to reporting quality in reviews with NMA (eg, PRISMA-NMA ${ }^{25}$ ).

- Articles identifying or addressing sources of bias and variation in NMA and published after PRISMA-NMA in 2014.

Study type 2 will meet any of these inclusion criterion

- Articles assessing the methodological quality (or risk of bias) of reviews with NMA (ie, a sample of NMAs are assessed for methodological quality; e.g. Chambers $2015^{35}$ ) using criteria that focus specifically on aspects of NMA not just on general aspects of systematic reviews.

We will include articles with any publication status and in any language, and where the coauthors are not fluent in the language, Google Translate will be used.

If through our main search, we identify a systematic review encompassing the eligible articles, or one aspect of the eligible article, we will use the results of the systematic review and only include primary studies published subsequent to the systematic review. For example, a review by Laws et al in $2019^{5}$ identified all guidance documents for conducting an NMA from countries throughout the world. We therefore would not search for guidance documents published before the last search date of this review. 


\section{Search strategy}

We will search Ovid MEDLINE (January 1946 to June 2020), the Cochrane library as well as the following grey literature databases: the EQUATOR Network (http:// www.equator-network.org/reportingguidelines/), Dissertation Abstracts, websites of evidence synthesis organisations (Campbell Collaboration Cochrane Multiple Treatments Methods Group, CADTH, NICE-DSU, Health Technology Assessment International (HTAi), Pharmaceutical Benefits Advisory Committee, Institut für Qualität und Wirtschaftlichkeit im Gesundheitswesen, European Network for Health Technology Assessment, Guidelines International Network, ISPOR, International Network of Agencies for Health Technology Assessment, and JBI) as well as methods collections (ie, Cochrane Methodology Register, AHRQ Effective Healthcare Programme). We will validate the MEDLINE strategy by using the PubMed IDs of 10 included studies (identified by experts prior to our eligibility screening) and evaluating whether the strategy identified the PMIDs (online supplemental appendix 2).

A systematic search strategy will be developed by two methodologists (CL and PW) without limitations to publication type, status, language or date to identify existing tools or articles. An information specialist will check the search strategy for MEDLINE Ovid and assess it using the PRESS (Peer Review Electronic Search Strategies) guidance. $^{36}$ The full search strategies for all databases and websites can be found in Appendix 2. To identify other potentially relevant studies, we will examine the reference lists of included studies. We will ask experts in methods for NMA to identify articles missed by our search. We will contact authors of abstracts to retrieve the full report or poster.

We will search the reference section of a bibliometric study of reviews with NMAs ${ }^{37}$ and extract the name of the journals that publish NMAs. We will then contact their editors in chief and ask if they have any in-house editorial standards for reviews with NMA.

\section{Process for screening, data extraction and analysis}

The eligibility criteria will be piloted in Microsoft Excel by two reviewers independently on a sample of 25 citations retrieved from the search to ensure consistent application. After high agreement $(>70 \%)$ is achieved, the Covidence $^{38}$ web-based tool (https://www.covidence.org) will be used by two reviewers to independently screen the citations based on the eligibility criteria. Disagreements will be discussed until consensus is reached. A third reviewer (CL) will arbitrate if disagreements cannot be resolved.

The data extraction form will be piloted by reviewers independently on a sample of five included papers to ensure consistent coding. Two independent authors will extract data on the characteristics of the studies and items. Any disagreements will be arbitrated by a third author.

\section{Data extraction}

The sources will first be categorised by the type of article coded as per our inclusion criteria. A table of tool characteristics will be developed with the following headings: first author, year; type of tool (tool, scale, checklist or domain-based tool); whether the tool is designed specific topic areas (specify); number of items; domains within the tool; whether the item relates to reporting or methodological quality (or other concepts such as precision, acceptability); how items and domains within the tool are rated; methods used to develop the tool (eg, review of items, Delphi study, expert consensus meeting) and the availability of an 'explanation and elaboration'. '

Data will be extracted on items that are potentially relevant to the risk of bias or quality of reviews with NMAs. Items will be initially extracted verbatim.

\section{Data analysis}

The following steps will be used when analysing items:

\section{Map to ROBIS domains}

Items will be mapped to ROBIS domains (study eligibility criteria; identification and selection of studies; data collection and study appraisal; and synthesis and findings) and specific items within the domains. The rationale for mapping items to ROBIS is that it is the only tool to assess risk of bias in reviews. Items that do not clearly map to the existing ROBIS domains will be listed separately and grouped by similar concept. New domains may be created if items do not fit well into the established ROBIS domains.

2. Split items so that each item only covers a single concept

Two or more concepts grouped in one item will be split so that each item covers a single concept. A rationale as to why the item was split will be described. For example, PRISMA-NMA item 15 ('Specify any assessment of risk of bias that may affect the cumulative evidence (eg, publication bias, selective reporting within studies)') will be split into two items because this item is represented by two items in ROBIS in the synthesis and findings domain, namely ' 4.5 Were the findings robust, for example, as demonstrated through funnel plot or sensitivity analyses?' and '4.6 Were biases in primary studies minimal or addressed in the synthesis?",

3. Group similar items

Items that are conceptually similar will be grouped together and noted with the source. We will classify items as relating to bias or other aspect of quality (eg, reporting). When relevant, items related to reporting will be reworded into items related to bias in NMA review conclusions.

4. Omit duplicate items (but keep these in a column in the table for transparency)

If items are worded vaguely or are unexplained, we will use an iterative process to interpret the item and ensure that there is a mutual understanding of the item between authors when coding. The process will be iterative, and 
if any gaps in items related to bias in reviews of NMA are identified, a new item will be inferred.

The final list of items deemed unique will be retained. We will reword items as signalling questions, where an answer of 'yes' suggests the absence of bias. We will provide examples to illustrate the items and write a rationale and description of each item. These items will be submitted in a multiround Delphi exercise by NMA experts who will give their opinion about each item's potential inclusion in the tool.

We will count the number of sources and unique items included. We will summarise the characteristics of included tools in tables and figures. We will calculate the median and IQR of items across all tools and tabulate the frequency of different biases identified in the tools.

\section{Patient and public involvement}

Patients or the public were not involved in the design of our research protocol.

\section{ETHICS AND DISSEMINATION}

No ethics approval was required as no human subjects were involved. Our research aims to develop a list of items related to bias in the goal of developing the first tool for assessing risk of bias in the findings of reviews with NMA. We plan to publish the full study open access in a peerreviewed journal, and disseminate the findings via social media (Twitter, Facebook and author affiliated websites).

Patients, healthcare providers and policy-makers need the highest quality evidence to make decisions about which treatments should be used in healthcare practice. Being able to critically appraise the findings of reviews with NMA is central to evidenced-based decision-making in patient care.

\footnotetext{
Author affiliations

${ }^{1}$ Knowledge Translation Program, Li Ka Shing Knowledge Institute of St Michael's Hospital, Toronto, Ontario, Canada

${ }^{2}$ Dalla Lana School of Public Health \& Institute of Health Policy, Management, and Evaluation, University of Toronto, University of Toronto, Toronto, Ontario, Canada ${ }^{3}$ Queen's Collaboration for Health Care Quality Joanna Briggs Institute Centre of Excellence, Queen's University, Kingston, Ontario, Canada

${ }^{4}$ School of Education, University of Ioannina, Ioannina, Greece

${ }^{5}$ Centre for Reviews and Dissemination, University of York, York, UK

${ }^{6}$ Ottawa Hospital Research Institute, Ottawa, Ontario, Canada

${ }^{7}$ School of Epidemiology and Public Health, Ottawa University, Ottawa, Ontario, Canada

${ }^{8}$ Institute of Social and Preventive Medicine, University of Bern, Bern, Switzerland ${ }^{9}$ Anesthesiology, Pharmacology \& Therapeutics, Cochrane Hypertension Review Group and the Therapeutics Initiative, University of British Columbia, Vancouver, BC, Canada

${ }^{10}$ MRC Clinical Trials Unit, University College London, London, UK

${ }^{11}$ Population Health Sciences, Bristol Medical School, University of Bristol, Bristol, UK
}

\section{Twitter Carole Lunny @carole_lunny}

Contributors $\mathrm{CL}$ conceived of the study; all authors contributed to the design the study; CL wrote the draft manuscript; CL, PW, ACT, BH, SD, GS, A-AV, IW and JW revised the manuscript; all authors edited the manuscript; and all authors read and approved the final manuscript.
Funding We have received a CIHR Spring Project Grant (ID 433402) for \$360 116 for this project (https://webapps.cihr-irsc.gc.ca/decisions/p/project_details. $\mathrm{html}$ ?applld=433402\&lang=en). The funders played no role in the conduct of this project. Andrea Tricco currently holds a Tier 2 Canada Research Chair in Knowledge Synthesis. Brian Hutton has previously received honoraria from Eversana Incorporated for the provision of methodologic advice related to the conduct of systematic reviews and meta-analysis.

Competing interests None declared.

Patient consent for publication Not required.

Provenance and peer review Not commissioned; externally peer reviewed.

Supplemental material This content has been supplied by the author(s). It has not been vetted by BMJ Publishing Group Limited (BMJ) and may not have been peer-reviewed. Any opinions or recommendations discussed are solely those of the author(s) and are not endorsed by BMJ. BMJ disclaims all liability and responsibility arising from any reliance placed on the content. Where the content includes any translated material, BMJ does not warrant the accuracy and reliability of the translations (including but not limited to local regulations, clinical guidelines, terminology, drug names and drug dosages), and is not responsible for any error and/or omissions arising from translation and adaptation or otherwise.

Open access This is an open access article distributed in accordance with the Creative Commons Attribution Non Commercial (CC BY-NC 4.0) license, which permits others to distribute, remix, adapt, build upon this work non-commercially, and license their derivative works on different terms, provided the original work is properly cited, appropriate credit is given, any changes made indicated, and the use is non-commercial. See: http://creativecommons.org/licenses/by-nc/4.0/.

\section{ORCID iDs}

Carole Lunny http://orcid.org/0000-0002-7825-6765

Andrea C Tricco http://orcid.org/0000-0002-4114-8971

Sofia Dias http://orcid.org/0000-0002-2172-0221

Brian Hutton http://orcid.org/0000-0001-5662-8647

\section{REFERENCES}

1 Ioannidis JPA. Integration of evidence from multiple meta-analyses: a primer on umbrella reviews, treatment networks and multiple treatments meta-analyses. CMAJ 2009;181:488-93.

2 Li L, Catalá-López F, Alonso-Arroyo A, et al. The global research collaboration of network meta-analysis: a social network analysis. PLoS One 2016;11:e0163239.

3 Kanters S, Ford N, Druyts E, et al. Use of network meta-analysis in clinical guidelines. Bull World Health Organ 2016;94:782-4.

4 Eiring Øystein, Brurberg KG, Nytrøen K, et al. Rapid methods including network meta-analysis to produce evidence in clinical decision support: a decision analysis. Syst Rev 2018;7:168.

5 Laws A, Tao R, Wang S, et al. A comparison of national guidelines for network meta-analysis. Value Health 2019;22:1178-86.

6 Egger M, Davey Smith G, Schneider M, et al. Bias in meta-analysis detected by a simple, graphical test. BMJ 1997;315:629-34.

7 Page MJ, McKenzie JE, Higgins JPT. Tools for assessing risk of reporting biases in studies and syntheses of studies: a systematic review. BMJ Open 2018;8:e019703.

8 Page MJ, McKenzie JE, Kirkham J, et al. Bias due to selective inclusion and reporting of outcomes and analyses in systematic reviews of randomised trials of healthcare interventions. Cochrane Database Syst Rev 2014:MR000035.

9 Petticrew M. Why certain systematic reviews reach uncertain conclusions. BMJ 2003;326:756-8.

10 Greco T, Biondi-Zoccai G, Saleh O, et al. The attractiveness of network meta-analysis: a comprehensive systematic and narrative review. Heart Lung Vessel 2015;7:133.

11 Jansen JP, Naci H. Is network meta-analysis as valid as standard pairwise meta-analysis? It all depends on the distribution of effect modifiers. BMC Med 2013;11:159.

$12 \mathrm{Li} \mathrm{T}$, Puhan MA, Vedula SS, et al. Network meta-analysis-highly attractive but more methodological research is needed. BMC Med 2011;9:79.

13 Dechartres A, Altman DG, Trinquart L, et al. Association between analytic strategy and estimates of treatment outcomes in metaanalyses. JAMA 2014;312:623-30.

14 Hart B, Lundh A, Bero L. Effect of reporting bias on meta-analyses of drug trials: reanalysis of meta-analyses. BMJ 2012;344:d7202.

15 Pham Ba', Klassen TP, Lawson ML, et al. Language of publication restrictions in systematic reviews gave different results depending on 
whether the intervention was conventional or complementary. J Clin Epidemiol 2005;58:769-76.

16 Mhaskar R, Emmanuel P, Mishra S, et al. Critical appraisal skills are essential to informed decision-making. Indian J Sex Transm Dis AIDS 2009;30:112

17 Whiting P. ROBIS: tool to assess risk of bias in systematic reviewsGuidance on how to use ROBIS, 2016. Available: http://www.bristol. ac.uk/media-library/sites/social-community-medicine/robis/robisgui dancedocument. pdf [Accessed 26 Mar 2018].

18 Canadian Institutes of Health Research. Knowledge user engagement; 2016.

19 Lunny C, Brennan SE, McDonald S, et al. Toward a comprehensive evidence map of overview of systematic review methods: paper 2risk of bias assessment; synthesis, presentation and summary of the findings; and assessment of the certainty of the evidence. Syst Rev 2018;7:159.

20 Whiting Pet al. Chapter 4. Phase 2: Review of existing quality assessment tools for systematic reviews. In: Evidence to inform the development of ROBIS, a new tool to assess the risk of bias in systematic reviews, 2013: p. 22-35. http://www.robis-tool.info

21 Shea BJ, Grimshaw JM, Wells GA, et al. Development of AMSTAR: a measurement tool to assess the methodological quality of systematic reviews. BMC Med Res Methodol 2007;7:10.

22 Oxman AD, Guyatt GH. Validation of an index of the quality of review articles. J Clin Epidemiol 1991;44:1271-8.

23 Pieper D, Buechter $R$, Jerinic $P$, et al. Overviews of reviews often have limited rigor: a systematic review. J Clin Epidemiol 2012;65:1267-73.

24 Shea BJ, Reeves BC, Wells G, et al. AMSTAR 2: a critical appraisal tool for systematic reviews that include randomised or nonrandomised studies of healthcare interventions, or both. BMJ 2017;358:j4008.

25 Hutton B, Catalá-López F, Moher D. [The PRISMA statement extension for systematic reviews incorporating network metaanalysis: PRISMA-NMA]. Med Clin 2016;147:262-6.

26 Dias S. NICE DSU technical support document 4: inconsistency in networks of evidence based on randomised controlled trials. London: National Institute for Health and Care Excellence (NICE), 2014.

27 Jansen JP, Trikalinos T, Cappelleri JC, et al. Indirect treatment comparison/network meta-analysis study questionnaire to assess relevance and credibility to inform health care decision making: an ISPOR-AMCP-NPC good practice Task force report. Value Health 2014;17:157-73.

28 Moher D, Schulz KF, Simera I, et al. Guidance for developers of health research reporting guidelines. PLoS Med 2010;7:e1000217.
29 Whiting P, Wolff R, Mallett S, et al. A proposed framework for developing quality assessment tools. Syst Rev 2017;6:204.

30 Sanderson S, Tatt ID, Higgins JPT. Tools for assessing quality and susceptibility to bias in observational studies in epidemiology: a systematic review and annotated bibliography. Int J Epidemiol 2007;36:666-76

31 Moher D, Shamseer L, Clarke M, et al. Preferred reporting items for systematic review and meta-analysis protocols (PRISMA-P) 2015 statement. Syst Rev 2015;4:p. 1.

32 Higgins JPet al. Cochrane Handbook for systematic reviews of interventions. John Wiley \& Sons, 2019.

33 Dias Set al. Chapter 8 Validity of network meta-analyses. In: Network meta-analysis for decision-making. John Wiley \& Sons, 2018.

34 Chandler Jet al. Methodological standards for the conduct of new Cochrane intervention reviews. SI: Cochrane Collaboration, 2013.

35 Chambers JD, Naci H, Wouters OJ, et al. An assessment of the methodological quality of published network meta-analyses: a systematic review. PLoS One 2015;10:e0121715.

36 McGowan J, Sampson M, Salzwedel DM, et al. PRESS Peer Review of Electronic Search Strategies: 2015 Guideline Statement. J Clin Epidemiol 2016;75:40-6.

37 Ban JK, Tadrous M, Lu AX, et al. History and publication trends in the diffusion and early uptake of indirect comparison meta-analytic methods to study drugs: animated coauthorship networks over time. BMJ Open 2018;8:e019110.

38 Veritas Health Innovation Melbourne. Covidence systematic review software; 2016

39 Guyatt G, Oxman AD, Akl EA, et al. Grade guidelines: 1. IntroductionGRADE evidence profiles and summary of findings tables. J Clin Epidemiol 2011;64:383-94.

40 Puhan MA, Schünemann $\mathrm{HJ}$, Murad $\mathrm{MH}$, et al. A grade Working group approach for rating the quality of treatment effect estimates from network meta-analysis. BMJ 2014;349:g5630.

41 Brignardello-Petersen R, Bonner A, Alexander PE, et al. Advances in the GRADE approach to rate the certainty in estimates from a network meta-analysis. J Clin Epidemiol 2018;93:36-44.

42 Nikolakopoulou A, Higgins JPT, Papakonstantinou T, et al. Cinema: an approach for assessing confidence in the results of a network meta-analysis. PLoS Med 2020;17:e1003082.

43 Phillippo DM, Dias S, Welton NJ, et al. Threshold analysis as an alternative to grade for assessing confidence in guideline recommendations based on network meta-analyses. Ann Intern Med 2019;170:538-46.

44 Moher D, Liberati A, Tetzlaff J, et al. Preferred reporting items for systematic reviews and meta-analyses: the PRISMA statement. PLoS Med 2009;6:e1000097. 